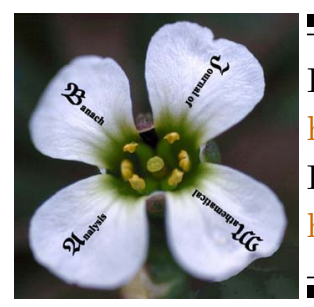

Banach J. Math. Anal. 10 (2016), no. 1, 147-168

http://dx.doi.org/10.1215/17358787-3345071

ISSN: $1735-8787$ (electronic)

http://projecteuclid.org/bjma

\title{
LINEAR AND NONLINEAR DEGENERATE ABSTRACT DIFFERENTIAL EQUATIONS WITH SMALL PARAMETER
}

\author{
VELI B. SHAKHMUROV ${ }^{1,2}$ \\ Communicated by L. P. Castro
}

\begin{abstract}
The boundary value problems for linear and nonlinear regular degenerate abstract differential equations are studied. The equations have the principal variable coefficients and a small parameter. The linear problem is considered on a parameter-dependent domain (i.e., on a moving domain). The maximal regularity properties of linear problems and the optimal regularity of the nonlinear problem are obtained. In application, the well-posedness of the Cauchy problem for degenerate parabolic equations and boundary value problems for degenerate anisotropic differential equations are established.
\end{abstract}

\section{IntroduCtion, NOTATION, AND BACKGROUND}

Boundary value problems (BVPs) for abstract differential equations (ADEs) have been studied extensively by many researchers (see [1], [3], [4], [8]-[21], and [24] and the references therein). A comprehensive introduction to ADEs and historical references may be found in [13] and [24]. The maximal regularity properties of ADEs have been studied in [1], [3]-[5], [8], [9], [16]-[20], and [23], for example. The main objective of the present article is to discuss the BVP for degenerate linear ADEs with variable coefficients

Copyright 2016 by the Tusi Mathematical Research Group.

Received Feb. 2, 2015; Accepted May 6, 2015.

2010 Mathematics Subject Classification. Primary 35J25; Secondary 35B65, 47N20.

Keywords. differential equations, semigroups of operators, Banach-valued function spaces, separable differential operators, operator-valued Fourier multipliers. 


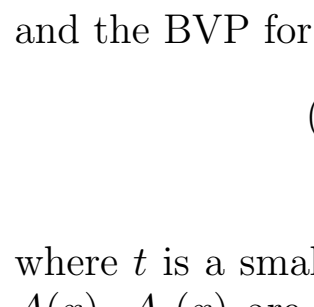

Banach J. Math. Anal. 10 (2016), no. 1, 147-168

http://dx.doi.org/10.1215/17358787-3345071

ISSN: $1735-8787$ (electronic)

http://projecteuclid.org/bjma

\title{
LINEAR AND NONLINEAR DEGENERATE ABSTRACT DIFFERENTIAL EQUATIONS WITH SMALL PARAMETER
}

\author{
VELI B. SHAKHMUROV ${ }^{1,2}$ \\ Communicated by L. P. Castro
}

\begin{abstract}
The boundary value problems for linear and nonlinear regular degenerate abstract differential equations are studied. The equations have the principal variable coefficients and a small parameter. The linear problem is considered on a parameter-dependent domain (i.e., on a moving domain). The maximal regularity properties of linear problems and the optimal regularity of the nonlinear problem are obtained. In application, the well-posedness of the Cauchy problem for degenerate parabolic equations and boundary value problems for degenerate anisotropic differential equations are established.
\end{abstract}

\section{IntroduCtion, NOTATION, AND BACKGROUND}

Boundary value problems (BVPs) for abstract differential equations (ADEs) have been studied extensively by many researchers (see [1], [3], [4], [8]-[21], and [24] and the references therein). A comprehensive introduction to ADEs and historical references may be found in [13] and [24]. The maximal regularity properties of ADEs have been studied in [1], [3]-[5], [8], [9], [16]-[20], and [23], for example. The main objective of the present article is to discuss the BVP for degenerate linear ADEs with variable coefficients

Copyright 2016 by the Tusi Mathematical Research Group.

Received Feb. 2, 2015; Accepted May 6, 2015.

2010 Mathematics Subject Classification. Primary 35J25; Secondary 35B65, 47N20.

Keywords. differential equations, semigroups of operators, Banach-valued function spaces, separable differential operators, operator-valued Fourier multipliers. 


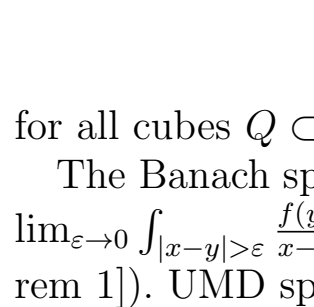

Banach J. Math. Anal. 10 (2016), no. 1, 147-168

http://dx.doi.org/10.1215/17358787-3345071

ISSN: $1735-8787$ (electronic)

http://projecteuclid.org/bjma

\title{
LINEAR AND NONLINEAR DEGENERATE ABSTRACT DIFFERENTIAL EQUATIONS WITH SMALL PARAMETER
}

\author{
VELI B. SHAKHMUROV ${ }^{1,2}$ \\ Communicated by L. P. Castro
}

\begin{abstract}
The boundary value problems for linear and nonlinear regular degenerate abstract differential equations are studied. The equations have the principal variable coefficients and a small parameter. The linear problem is considered on a parameter-dependent domain (i.e., on a moving domain). The maximal regularity properties of linear problems and the optimal regularity of the nonlinear problem are obtained. In application, the well-posedness of the Cauchy problem for degenerate parabolic equations and boundary value problems for degenerate anisotropic differential equations are established.
\end{abstract}

\section{IntroduCtion, NOTATION, AND BACKGROUND}

Boundary value problems (BVPs) for abstract differential equations (ADEs) have been studied extensively by many researchers (see [1], [3], [4], [8]-[21], and [24] and the references therein). A comprehensive introduction to ADEs and historical references may be found in [13] and [24]. The maximal regularity properties of ADEs have been studied in [1], [3]-[5], [8], [9], [16]-[20], and [23], for example. The main objective of the present article is to discuss the BVP for degenerate linear ADEs with variable coefficients

Copyright 2016 by the Tusi Mathematical Research Group.

Received Feb. 2, 2015; Accepted May 6, 2015.

2010 Mathematics Subject Classification. Primary 35J25; Secondary 35B65, 47N20.

Keywords. differential equations, semigroups of operators, Banach-valued function spaces, separable differential operators, operator-valued Fourier multipliers. 


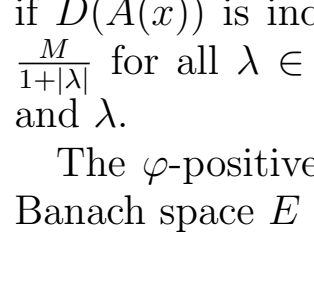

Banach J. Math. Anal. 10 (2016), no. 1, 147-168

http://dx.doi.org/10.1215/17358787-3345071

ISSN: $1735-8787$ (electronic)

http://projecteuclid.org/bjma

\title{
LINEAR AND NONLINEAR DEGENERATE ABSTRACT DIFFERENTIAL EQUATIONS WITH SMALL PARAMETER
}

\author{
VELI B. SHAKHMUROV ${ }^{1,2}$ \\ Communicated by L. P. Castro
}

\begin{abstract}
The boundary value problems for linear and nonlinear regular degenerate abstract differential equations are studied. The equations have the principal variable coefficients and a small parameter. The linear problem is considered on a parameter-dependent domain (i.e., on a moving domain). The maximal regularity properties of linear problems and the optimal regularity of the nonlinear problem are obtained. In application, the well-posedness of the Cauchy problem for degenerate parabolic equations and boundary value problems for degenerate anisotropic differential equations are established.
\end{abstract}

\section{IntroduCtion, NOTATION, AND BACKGROUND}

Boundary value problems (BVPs) for abstract differential equations (ADEs) have been studied extensively by many researchers (see [1], [3], [4], [8]-[21], and [24] and the references therein). A comprehensive introduction to ADEs and historical references may be found in [13] and [24]. The maximal regularity properties of ADEs have been studied in [1], [3]-[5], [8], [9], [16]-[20], and [23], for example. The main objective of the present article is to discuss the BVP for degenerate linear ADEs with variable coefficients

Copyright 2016 by the Tusi Mathematical Research Group.

Received Feb. 2, 2015; Accepted May 6, 2015.

2010 Mathematics Subject Classification. Primary 35J25; Secondary 35B65, 47N20.

Keywords. differential equations, semigroups of operators, Banach-valued function spaces, separable differential operators, operator-valued Fourier multipliers. 


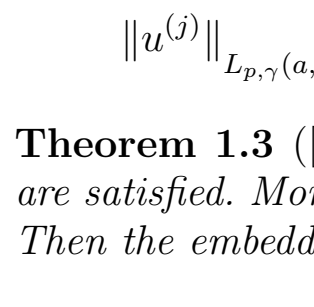

Banach J. Math. Anal. 10 (2016), no. 1, 147-168

http://dx.doi.org/10.1215/17358787-3345071

ISSN: $1735-8787$ (electronic)

http://projecteuclid.org/bjma

\title{
LINEAR AND NONLINEAR DEGENERATE ABSTRACT DIFFERENTIAL EQUATIONS WITH SMALL PARAMETER
}

\author{
VELI B. SHAKHMUROV ${ }^{1,2}$ \\ Communicated by L. P. Castro
}

\begin{abstract}
The boundary value problems for linear and nonlinear regular degenerate abstract differential equations are studied. The equations have the principal variable coefficients and a small parameter. The linear problem is considered on a parameter-dependent domain (i.e., on a moving domain). The maximal regularity properties of linear problems and the optimal regularity of the nonlinear problem are obtained. In application, the well-posedness of the Cauchy problem for degenerate parabolic equations and boundary value problems for degenerate anisotropic differential equations are established.
\end{abstract}

\section{IntroduCtion, NOTATION, AND BACKGROUND}

Boundary value problems (BVPs) for abstract differential equations (ADEs) have been studied extensively by many researchers (see [1], [3], [4], [8]-[21], and [24] and the references therein). A comprehensive introduction to ADEs and historical references may be found in [13] and [24]. The maximal regularity properties of ADEs have been studied in [1], [3]-[5], [8], [9], [16]-[20], and [23], for example. The main objective of the present article is to discuss the BVP for degenerate linear ADEs with variable coefficients

Copyright 2016 by the Tusi Mathematical Research Group.

Received Feb. 2, 2015; Accepted May 6, 2015.

2010 Mathematics Subject Classification. Primary 35J25; Secondary 35B65, 47N20.

Keywords. differential equations, semigroups of operators, Banach-valued function spaces, separable differential operators, operator-valued Fourier multipliers. 


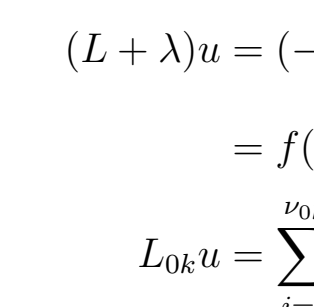

Banach J. Math. Anal. 10 (2016), no. 1, 147-168

http://dx.doi.org/10.1215/17358787-3345071

ISSN: $1735-8787$ (electronic)

http://projecteuclid.org/bjma

\title{
LINEAR AND NONLINEAR DEGENERATE ABSTRACT DIFFERENTIAL EQUATIONS WITH SMALL PARAMETER
}

\author{
VELI B. SHAKHMUROV ${ }^{1,2}$ \\ Communicated by L. P. Castro
}

\begin{abstract}
The boundary value problems for linear and nonlinear regular degenerate abstract differential equations are studied. The equations have the principal variable coefficients and a small parameter. The linear problem is considered on a parameter-dependent domain (i.e., on a moving domain). The maximal regularity properties of linear problems and the optimal regularity of the nonlinear problem are obtained. In application, the well-posedness of the Cauchy problem for degenerate parabolic equations and boundary value problems for degenerate anisotropic differential equations are established.
\end{abstract}

\section{IntroduCtion, NOTATION, AND BACKGROUND}

Boundary value problems (BVPs) for abstract differential equations (ADEs) have been studied extensively by many researchers (see [1], [3], [4], [8]-[21], and [24] and the references therein). A comprehensive introduction to ADEs and historical references may be found in [13] and [24]. The maximal regularity properties of ADEs have been studied in [1], [3]-[5], [8], [9], [16]-[20], and [23], for example. The main objective of the present article is to discuss the BVP for degenerate linear ADEs with variable coefficients

Copyright 2016 by the Tusi Mathematical Research Group.

Received Feb. 2, 2015; Accepted May 6, 2015.

2010 Mathematics Subject Classification. Primary 35J25; Secondary 35B65, 47N20.

Keywords. differential equations, semigroups of operators, Banach-valued function spaces, separable differential operators, operator-valued Fourier multipliers. 


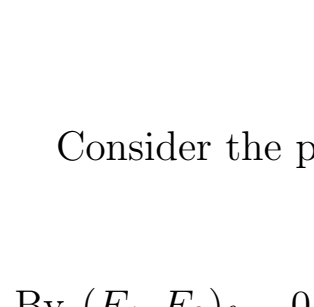

Banach J. Math. Anal. 10 (2016), no. 1, 147-168

http://dx.doi.org/10.1215/17358787-3345071

ISSN: $1735-8787$ (electronic)

http://projecteuclid.org/bjma

\title{
LINEAR AND NONLINEAR DEGENERATE ABSTRACT DIFFERENTIAL EQUATIONS WITH SMALL PARAMETER
}

\author{
VELI B. SHAKHMUROV ${ }^{1,2}$ \\ Communicated by L. P. Castro
}

\begin{abstract}
The boundary value problems for linear and nonlinear regular degenerate abstract differential equations are studied. The equations have the principal variable coefficients and a small parameter. The linear problem is considered on a parameter-dependent domain (i.e., on a moving domain). The maximal regularity properties of linear problems and the optimal regularity of the nonlinear problem are obtained. In application, the well-posedness of the Cauchy problem for degenerate parabolic equations and boundary value problems for degenerate anisotropic differential equations are established.
\end{abstract}

\section{IntroduCtion, NOTATION, AND BACKGROUND}

Boundary value problems (BVPs) for abstract differential equations (ADEs) have been studied extensively by many researchers (see [1], [3], [4], [8]-[21], and [24] and the references therein). A comprehensive introduction to ADEs and historical references may be found in [13] and [24]. The maximal regularity properties of ADEs have been studied in [1], [3]-[5], [8], [9], [16]-[20], and [23], for example. The main objective of the present article is to discuss the BVP for degenerate linear ADEs with variable coefficients

Copyright 2016 by the Tusi Mathematical Research Group.

Received Feb. 2, 2015; Accepted May 6, 2015.

2010 Mathematics Subject Classification. Primary 35J25; Secondary 35B65, 47N20.

Keywords. differential equations, semigroups of operators, Banach-valued function spaces, separable differential operators, operator-valued Fourier multipliers. 


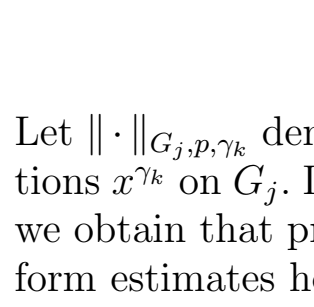

Banach J. Math. Anal. 10 (2016), no. 1, 147-168

http://dx.doi.org/10.1215/17358787-3345071

ISSN: $1735-8787$ (electronic)

http://projecteuclid.org/bjma

\title{
LINEAR AND NONLINEAR DEGENERATE ABSTRACT DIFFERENTIAL EQUATIONS WITH SMALL PARAMETER
}

\author{
VELI B. SHAKHMUROV ${ }^{1,2}$ \\ Communicated by L. P. Castro
}

\begin{abstract}
The boundary value problems for linear and nonlinear regular degenerate abstract differential equations are studied. The equations have the principal variable coefficients and a small parameter. The linear problem is considered on a parameter-dependent domain (i.e., on a moving domain). The maximal regularity properties of linear problems and the optimal regularity of the nonlinear problem are obtained. In application, the well-posedness of the Cauchy problem for degenerate parabolic equations and boundary value problems for degenerate anisotropic differential equations are established.
\end{abstract}

\section{IntroduCtion, NOTATION, AND BACKGROUND}

Boundary value problems (BVPs) for abstract differential equations (ADEs) have been studied extensively by many researchers (see [1], [3], [4], [8]-[21], and [24] and the references therein). A comprehensive introduction to ADEs and historical references may be found in [13] and [24]. The maximal regularity properties of ADEs have been studied in [1], [3]-[5], [8], [9], [16]-[20], and [23], for example. The main objective of the present article is to discuss the BVP for degenerate linear ADEs with variable coefficients

Copyright 2016 by the Tusi Mathematical Research Group.

Received Feb. 2, 2015; Accepted May 6, 2015.

2010 Mathematics Subject Classification. Primary 35J25; Secondary 35B65, 47N20.

Keywords. differential equations, semigroups of operators, Banach-valued function spaces, separable differential operators, operator-valued Fourier multipliers. 


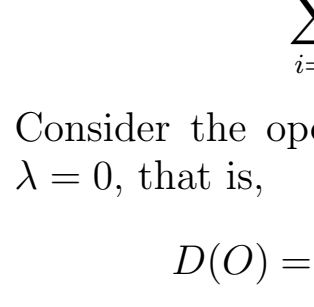

Banach J. Math. Anal. 10 (2016), no. 1, 147-168

http://dx.doi.org/10.1215/17358787-3345071

ISSN: $1735-8787$ (electronic)

http://projecteuclid.org/bjma

\title{
LINEAR AND NONLINEAR DEGENERATE ABSTRACT DIFFERENTIAL EQUATIONS WITH SMALL PARAMETER
}

\author{
VELI B. SHAKHMUROV ${ }^{1,2}$ \\ Communicated by L. P. Castro
}

\begin{abstract}
The boundary value problems for linear and nonlinear regular degenerate abstract differential equations are studied. The equations have the principal variable coefficients and a small parameter. The linear problem is considered on a parameter-dependent domain (i.e., on a moving domain). The maximal regularity properties of linear problems and the optimal regularity of the nonlinear problem are obtained. In application, the well-posedness of the Cauchy problem for degenerate parabolic equations and boundary value problems for degenerate anisotropic differential equations are established.
\end{abstract}

\section{IntroduCtion, NOTATION, AND BACKGROUND}

Boundary value problems (BVPs) for abstract differential equations (ADEs) have been studied extensively by many researchers (see [1], [3], [4], [8]-[21], and [24] and the references therein). A comprehensive introduction to ADEs and historical references may be found in [13] and [24]. The maximal regularity properties of ADEs have been studied in [1], [3]-[5], [8], [9], [16]-[20], and [23], for example. The main objective of the present article is to discuss the BVP for degenerate linear ADEs with variable coefficients

Copyright 2016 by the Tusi Mathematical Research Group.

Received Feb. 2, 2015; Accepted May 6, 2015.

2010 Mathematics Subject Classification. Primary 35J25; Secondary 35B65, 47N20.

Keywords. differential equations, semigroups of operators, Banach-valued function spaces, separable differential operators, operator-valued Fourier multipliers. 


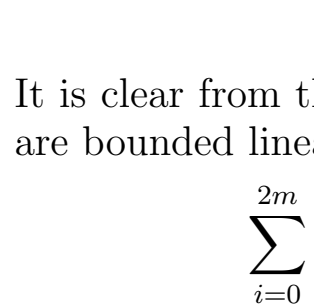

Banach J. Math. Anal. 10 (2016), no. 1, 147-168

http://dx.doi.org/10.1215/17358787-3345071

ISSN: $1735-8787$ (electronic)

http://projecteuclid.org/bjma

\title{
LINEAR AND NONLINEAR DEGENERATE ABSTRACT DIFFERENTIAL EQUATIONS WITH SMALL PARAMETER
}

\author{
VELI B. SHAKHMUROV ${ }^{1,2}$ \\ Communicated by L. P. Castro
}

\begin{abstract}
The boundary value problems for linear and nonlinear regular degenerate abstract differential equations are studied. The equations have the principal variable coefficients and a small parameter. The linear problem is considered on a parameter-dependent domain (i.e., on a moving domain). The maximal regularity properties of linear problems and the optimal regularity of the nonlinear problem are obtained. In application, the well-posedness of the Cauchy problem for degenerate parabolic equations and boundary value problems for degenerate anisotropic differential equations are established.
\end{abstract}

\section{IntroduCtion, NOTATION, AND BACKGROUND}

Boundary value problems (BVPs) for abstract differential equations (ADEs) have been studied extensively by many researchers (see [1], [3], [4], [8]-[21], and [24] and the references therein). A comprehensive introduction to ADEs and historical references may be found in [13] and [24]. The maximal regularity properties of ADEs have been studied in [1], [3]-[5], [8], [9], [16]-[20], and [23], for example. The main objective of the present article is to discuss the BVP for degenerate linear ADEs with variable coefficients

Copyright 2016 by the Tusi Mathematical Research Group.

Received Feb. 2, 2015; Accepted May 6, 2015.

2010 Mathematics Subject Classification. Primary 35J25; Secondary 35B65, 47N20.

Keywords. differential equations, semigroups of operators, Banach-valued function spaces, separable differential operators, operator-valued Fourier multipliers. 


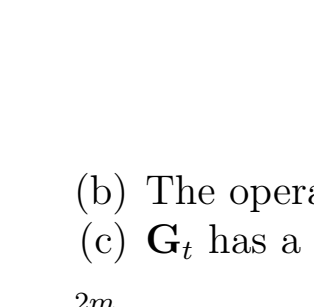

Banach J. Math. Anal. 10 (2016), no. 1, 147-168

http://dx.doi.org/10.1215/17358787-3345071

ISSN: $1735-8787$ (electronic)

http://projecteuclid.org/bjma

\title{
LINEAR AND NONLINEAR DEGENERATE ABSTRACT DIFFERENTIAL EQUATIONS WITH SMALL PARAMETER
}

\author{
VELI B. SHAKHMUROV ${ }^{1,2}$ \\ Communicated by L. P. Castro
}

\begin{abstract}
The boundary value problems for linear and nonlinear regular degenerate abstract differential equations are studied. The equations have the principal variable coefficients and a small parameter. The linear problem is considered on a parameter-dependent domain (i.e., on a moving domain). The maximal regularity properties of linear problems and the optimal regularity of the nonlinear problem are obtained. In application, the well-posedness of the Cauchy problem for degenerate parabolic equations and boundary value problems for degenerate anisotropic differential equations are established.
\end{abstract}

\section{IntroduCtion, NOTATION, AND BACKGROUND}

Boundary value problems (BVPs) for abstract differential equations (ADEs) have been studied extensively by many researchers (see [1], [3], [4], [8]-[21], and [24] and the references therein). A comprehensive introduction to ADEs and historical references may be found in [13] and [24]. The maximal regularity properties of ADEs have been studied in [1], [3]-[5], [8], [9], [16]-[20], and [23], for example. The main objective of the present article is to discuss the BVP for degenerate linear ADEs with variable coefficients

Copyright 2016 by the Tusi Mathematical Research Group.

Received Feb. 2, 2015; Accepted May 6, 2015.

2010 Mathematics Subject Classification. Primary 35J25; Secondary 35B65, 47N20.

Keywords. differential equations, semigroups of operators, Banach-valued function spaces, separable differential operators, operator-valued Fourier multipliers. 


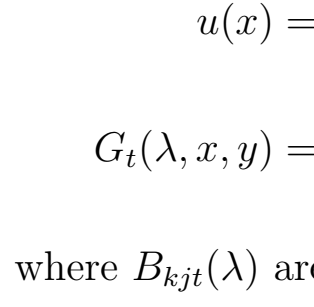

Banach J. Math. Anal. 10 (2016), no. 1, 147-168

http://dx.doi.org/10.1215/17358787-3345071

ISSN: $1735-8787$ (electronic)

http://projecteuclid.org/bjma

\title{
LINEAR AND NONLINEAR DEGENERATE ABSTRACT DIFFERENTIAL EQUATIONS WITH SMALL PARAMETER
}

\author{
VELI B. SHAKHMUROV ${ }^{1,2}$ \\ Communicated by L. P. Castro
}

\begin{abstract}
The boundary value problems for linear and nonlinear regular degenerate abstract differential equations are studied. The equations have the principal variable coefficients and a small parameter. The linear problem is considered on a parameter-dependent domain (i.e., on a moving domain). The maximal regularity properties of linear problems and the optimal regularity of the nonlinear problem are obtained. In application, the well-posedness of the Cauchy problem for degenerate parabolic equations and boundary value problems for degenerate anisotropic differential equations are established.
\end{abstract}

\section{IntroduCtion, NOTATION, AND BACKGROUND}

Boundary value problems (BVPs) for abstract differential equations (ADEs) have been studied extensively by many researchers (see [1], [3], [4], [8]-[21], and [24] and the references therein). A comprehensive introduction to ADEs and historical references may be found in [13] and [24]. The maximal regularity properties of ADEs have been studied in [1], [3]-[5], [8], [9], [16]-[20], and [23], for example. The main objective of the present article is to discuss the BVP for degenerate linear ADEs with variable coefficients

Copyright 2016 by the Tusi Mathematical Research Group.

Received Feb. 2, 2015; Accepted May 6, 2015.

2010 Mathematics Subject Classification. Primary 35J25; Secondary 35B65, 47N20.

Keywords. differential equations, semigroups of operators, Banach-valued function spaces, separable differential operators, operator-valued Fourier multipliers. 


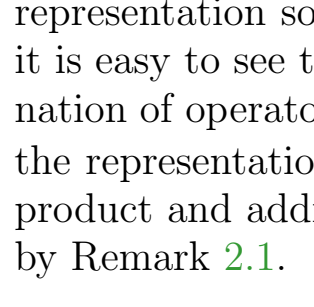

Banach J. Math. Anal. 10 (2016), no. 1, 147-168

http://dx.doi.org/10.1215/17358787-3345071

ISSN: $1735-8787$ (electronic)

http://projecteuclid.org/bjma

\title{
LINEAR AND NONLINEAR DEGENERATE ABSTRACT DIFFERENTIAL EQUATIONS WITH SMALL PARAMETER
}

\author{
VELI B. SHAKHMUROV ${ }^{1,2}$ \\ Communicated by L. P. Castro
}

\begin{abstract}
The boundary value problems for linear and nonlinear regular degenerate abstract differential equations are studied. The equations have the principal variable coefficients and a small parameter. The linear problem is considered on a parameter-dependent domain (i.e., on a moving domain). The maximal regularity properties of linear problems and the optimal regularity of the nonlinear problem are obtained. In application, the well-posedness of the Cauchy problem for degenerate parabolic equations and boundary value problems for degenerate anisotropic differential equations are established.
\end{abstract}

\section{IntroduCtion, NOTATION, AND BACKGROUND}

Boundary value problems (BVPs) for abstract differential equations (ADEs) have been studied extensively by many researchers (see [1], [3], [4], [8]-[21], and [24] and the references therein). A comprehensive introduction to ADEs and historical references may be found in [13] and [24]. The maximal regularity properties of ADEs have been studied in [1], [3]-[5], [8], [9], [16]-[20], and [23], for example. The main objective of the present article is to discuss the BVP for degenerate linear ADEs with variable coefficients

Copyright 2016 by the Tusi Mathematical Research Group.

Received Feb. 2, 2015; Accepted May 6, 2015.

2010 Mathematics Subject Classification. Primary 35J25; Secondary 35B65, 47N20.

Keywords. differential equations, semigroups of operators, Banach-valued function spaces, separable differential operators, operator-valued Fourier multipliers. 


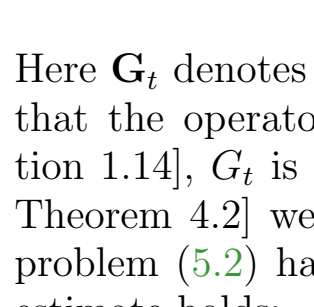

Banach J. Math. Anal. 10 (2016), no. 1, 147-168

http://dx.doi.org/10.1215/17358787-3345071

ISSN: $1735-8787$ (electronic)

http://projecteuclid.org/bjma

\title{
LINEAR AND NONLINEAR DEGENERATE ABSTRACT DIFFERENTIAL EQUATIONS WITH SMALL PARAMETER
}

\author{
VELI B. SHAKHMUROV ${ }^{1,2}$ \\ Communicated by L. P. Castro
}

\begin{abstract}
The boundary value problems for linear and nonlinear regular degenerate abstract differential equations are studied. The equations have the principal variable coefficients and a small parameter. The linear problem is considered on a parameter-dependent domain (i.e., on a moving domain). The maximal regularity properties of linear problems and the optimal regularity of the nonlinear problem are obtained. In application, the well-posedness of the Cauchy problem for degenerate parabolic equations and boundary value problems for degenerate anisotropic differential equations are established.
\end{abstract}

\section{IntroduCtion, NOTATION, AND BACKGROUND}

Boundary value problems (BVPs) for abstract differential equations (ADEs) have been studied extensively by many researchers (see [1], [3], [4], [8]-[21], and [24] and the references therein). A comprehensive introduction to ADEs and historical references may be found in [13] and [24]. The maximal regularity properties of ADEs have been studied in [1], [3]-[5], [8], [9], [16]-[20], and [23], for example. The main objective of the present article is to discuss the BVP for degenerate linear ADEs with variable coefficients

Copyright 2016 by the Tusi Mathematical Research Group.

Received Feb. 2, 2015; Accepted May 6, 2015.

2010 Mathematics Subject Classification. Primary 35J25; Secondary 35B65, 47N20.

Keywords. differential equations, semigroups of operators, Banach-valued function spaces, separable differential operators, operator-valued Fourier multipliers. 


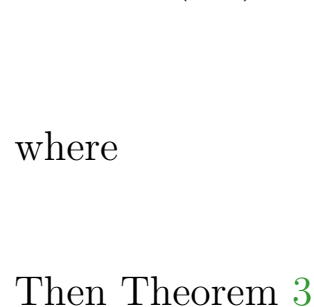

Banach J. Math. Anal. 10 (2016), no. 1, 147-168

http://dx.doi.org/10.1215/17358787-3345071

ISSN: $1735-8787$ (electronic)

http://projecteuclid.org/bjma

\title{
LINEAR AND NONLINEAR DEGENERATE ABSTRACT DIFFERENTIAL EQUATIONS WITH SMALL PARAMETER
}

\author{
VELI B. SHAKHMUROV ${ }^{1,2}$ \\ Communicated by L. P. Castro
}

\begin{abstract}
The boundary value problems for linear and nonlinear regular degenerate abstract differential equations are studied. The equations have the principal variable coefficients and a small parameter. The linear problem is considered on a parameter-dependent domain (i.e., on a moving domain). The maximal regularity properties of linear problems and the optimal regularity of the nonlinear problem are obtained. In application, the well-posedness of the Cauchy problem for degenerate parabolic equations and boundary value problems for degenerate anisotropic differential equations are established.
\end{abstract}

\section{IntroduCtion, NOTATION, AND BACKGROUND}

Boundary value problems (BVPs) for abstract differential equations (ADEs) have been studied extensively by many researchers (see [1], [3], [4], [8]-[21], and [24] and the references therein). A comprehensive introduction to ADEs and historical references may be found in [13] and [24]. The maximal regularity properties of ADEs have been studied in [1], [3]-[5], [8], [9], [16]-[20], and [23], for example. The main objective of the present article is to discuss the BVP for degenerate linear ADEs with variable coefficients

Copyright 2016 by the Tusi Mathematical Research Group.

Received Feb. 2, 2015; Accepted May 6, 2015.

2010 Mathematics Subject Classification. Primary 35J25; Secondary 35B65, 47N20.

Keywords. differential equations, semigroups of operators, Banach-valued function spaces, separable differential operators, operator-valued Fourier multipliers. 


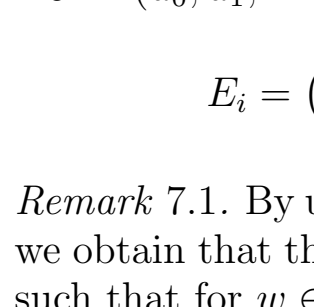

Banach J. Math. Anal. 10 (2016), no. 1, 147-168

http://dx.doi.org/10.1215/17358787-3345071

ISSN: $1735-8787$ (electronic)

http://projecteuclid.org/bjma

\title{
LINEAR AND NONLINEAR DEGENERATE ABSTRACT DIFFERENTIAL EQUATIONS WITH SMALL PARAMETER
}

\author{
VELI B. SHAKHMUROV ${ }^{1,2}$ \\ Communicated by L. P. Castro
}

\begin{abstract}
The boundary value problems for linear and nonlinear regular degenerate abstract differential equations are studied. The equations have the principal variable coefficients and a small parameter. The linear problem is considered on a parameter-dependent domain (i.e., on a moving domain). The maximal regularity properties of linear problems and the optimal regularity of the nonlinear problem are obtained. In application, the well-posedness of the Cauchy problem for degenerate parabolic equations and boundary value problems for degenerate anisotropic differential equations are established.
\end{abstract}

\section{IntroduCtion, NOTATION, AND BACKGROUND}

Boundary value problems (BVPs) for abstract differential equations (ADEs) have been studied extensively by many researchers (see [1], [3], [4], [8]-[21], and [24] and the references therein). A comprehensive introduction to ADEs and historical references may be found in [13] and [24]. The maximal regularity properties of ADEs have been studied in [1], [3]-[5], [8], [9], [16]-[20], and [23], for example. The main objective of the present article is to discuss the BVP for degenerate linear ADEs with variable coefficients

Copyright 2016 by the Tusi Mathematical Research Group.

Received Feb. 2, 2015; Accepted May 6, 2015.

2010 Mathematics Subject Classification. Primary 35J25; Secondary 35B65, 47N20.

Keywords. differential equations, semigroups of operators, Banach-valued function spaces, separable differential operators, operator-valued Fourier multipliers. 


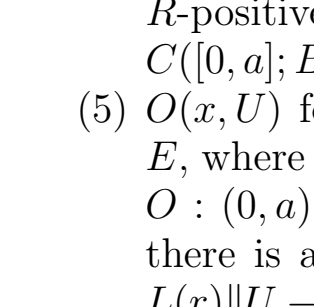

Banach J. Math. Anal. 10 (2016), no. 1, 147-168

http://dx.doi.org/10.1215/17358787-3345071

ISSN: $1735-8787$ (electronic)

http://projecteuclid.org/bjma

\title{
LINEAR AND NONLINEAR DEGENERATE ABSTRACT DIFFERENTIAL EQUATIONS WITH SMALL PARAMETER
}

\author{
VELI B. SHAKHMUROV ${ }^{1,2}$ \\ Communicated by L. P. Castro
}

\begin{abstract}
The boundary value problems for linear and nonlinear regular degenerate abstract differential equations are studied. The equations have the principal variable coefficients and a small parameter. The linear problem is considered on a parameter-dependent domain (i.e., on a moving domain). The maximal regularity properties of linear problems and the optimal regularity of the nonlinear problem are obtained. In application, the well-posedness of the Cauchy problem for degenerate parabolic equations and boundary value problems for degenerate anisotropic differential equations are established.
\end{abstract}

\section{IntroduCtion, NOTATION, AND BACKGROUND}

Boundary value problems (BVPs) for abstract differential equations (ADEs) have been studied extensively by many researchers (see [1], [3], [4], [8]-[21], and [24] and the references therein). A comprehensive introduction to ADEs and historical references may be found in [13] and [24]. The maximal regularity properties of ADEs have been studied in [1], [3]-[5], [8], [9], [16]-[20], and [23], for example. The main objective of the present article is to discuss the BVP for degenerate linear ADEs with variable coefficients

Copyright 2016 by the Tusi Mathematical Research Group.

Received Feb. 2, 2015; Accepted May 6, 2015.

2010 Mathematics Subject Classification. Primary 35J25; Secondary 35B65, 47N20.

Keywords. differential equations, semigroups of operators, Banach-valued function spaces, separable differential operators, operator-valued Fourier multipliers. 


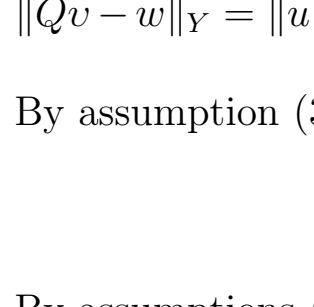

Banach J. Math. Anal. 10 (2016), no. 1, 147-168

http://dx.doi.org/10.1215/17358787-3345071

ISSN: $1735-8787$ (electronic)

http://projecteuclid.org/bjma

\title{
LINEAR AND NONLINEAR DEGENERATE ABSTRACT DIFFERENTIAL EQUATIONS WITH SMALL PARAMETER
}

\author{
VELI B. SHAKHMUROV ${ }^{1,2}$ \\ Communicated by L. P. Castro
}

\begin{abstract}
The boundary value problems for linear and nonlinear regular degenerate abstract differential equations are studied. The equations have the principal variable coefficients and a small parameter. The linear problem is considered on a parameter-dependent domain (i.e., on a moving domain). The maximal regularity properties of linear problems and the optimal regularity of the nonlinear problem are obtained. In application, the well-posedness of the Cauchy problem for degenerate parabolic equations and boundary value problems for degenerate anisotropic differential equations are established.
\end{abstract}

\section{IntroduCtion, NOTATION, AND BACKGROUND}

Boundary value problems (BVPs) for abstract differential equations (ADEs) have been studied extensively by many researchers (see [1], [3], [4], [8]-[21], and [24] and the references therein). A comprehensive introduction to ADEs and historical references may be found in [13] and [24]. The maximal regularity properties of ADEs have been studied in [1], [3]-[5], [8], [9], [16]-[20], and [23], for example. The main objective of the present article is to discuss the BVP for degenerate linear ADEs with variable coefficients

Copyright 2016 by the Tusi Mathematical Research Group.

Received Feb. 2, 2015; Accepted May 6, 2015.

2010 Mathematics Subject Classification. Primary 35J25; Secondary 35B65, 47N20.

Keywords. differential equations, semigroups of operators, Banach-valued function spaces, separable differential operators, operator-valued Fourier multipliers. 


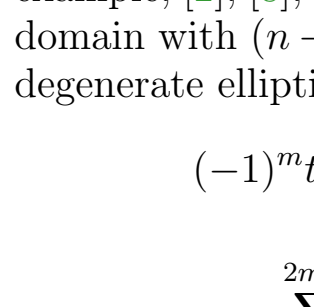

Banach J. Math. Anal. 10 (2016), no. 1, 147-168

http://dx.doi.org/10.1215/17358787-3345071

ISSN: $1735-8787$ (electronic)

http://projecteuclid.org/bjma

\title{
LINEAR AND NONLINEAR DEGENERATE ABSTRACT DIFFERENTIAL EQUATIONS WITH SMALL PARAMETER
}

\author{
VELI B. SHAKHMUROV ${ }^{1,2}$ \\ Communicated by L. P. Castro
}

\begin{abstract}
The boundary value problems for linear and nonlinear regular degenerate abstract differential equations are studied. The equations have the principal variable coefficients and a small parameter. The linear problem is considered on a parameter-dependent domain (i.e., on a moving domain). The maximal regularity properties of linear problems and the optimal regularity of the nonlinear problem are obtained. In application, the well-posedness of the Cauchy problem for degenerate parabolic equations and boundary value problems for degenerate anisotropic differential equations are established.
\end{abstract}

\section{IntroduCtion, NOTATION, AND BACKGROUND}

Boundary value problems (BVPs) for abstract differential equations (ADEs) have been studied extensively by many researchers (see [1], [3], [4], [8]-[21], and [24] and the references therein). A comprehensive introduction to ADEs and historical references may be found in [13] and [24]. The maximal regularity properties of ADEs have been studied in [1], [3]-[5], [8], [9], [16]-[20], and [23], for example. The main objective of the present article is to discuss the BVP for degenerate linear ADEs with variable coefficients

Copyright 2016 by the Tusi Mathematical Research Group.

Received Feb. 2, 2015; Accepted May 6, 2015.

2010 Mathematics Subject Classification. Primary 35J25; Secondary 35B65, 47N20.

Keywords. differential equations, semigroups of operators, Banach-valued function spaces, separable differential operators, operator-valued Fourier multipliers. 


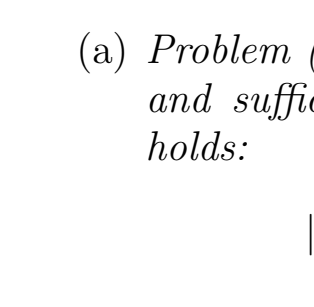

Banach J. Math. Anal. 10 (2016), no. 1, 147-168

http://dx.doi.org/10.1215/17358787-3345071

ISSN: $1735-8787$ (electronic)

http://projecteuclid.org/bjma

\title{
LINEAR AND NONLINEAR DEGENERATE ABSTRACT DIFFERENTIAL EQUATIONS WITH SMALL PARAMETER
}

\author{
VELI B. SHAKHMUROV ${ }^{1,2}$ \\ Communicated by L. P. Castro
}

\begin{abstract}
The boundary value problems for linear and nonlinear regular degenerate abstract differential equations are studied. The equations have the principal variable coefficients and a small parameter. The linear problem is considered on a parameter-dependent domain (i.e., on a moving domain). The maximal regularity properties of linear problems and the optimal regularity of the nonlinear problem are obtained. In application, the well-posedness of the Cauchy problem for degenerate parabolic equations and boundary value problems for degenerate anisotropic differential equations are established.
\end{abstract}

\section{IntroduCtion, NOTATION, AND BACKGROUND}

Boundary value problems (BVPs) for abstract differential equations (ADEs) have been studied extensively by many researchers (see [1], [3], [4], [8]-[21], and [24] and the references therein). A comprehensive introduction to ADEs and historical references may be found in [13] and [24]. The maximal regularity properties of ADEs have been studied in [1], [3]-[5], [8], [9], [16]-[20], and [23], for example. The main objective of the present article is to discuss the BVP for degenerate linear ADEs with variable coefficients

Copyright 2016 by the Tusi Mathematical Research Group.

Received Feb. 2, 2015; Accepted May 6, 2015.

2010 Mathematics Subject Classification. Primary 35J25; Secondary 35B65, 47N20.

Keywords. differential equations, semigroups of operators, Banach-valued function spaces, separable differential operators, operator-valued Fourier multipliers. 


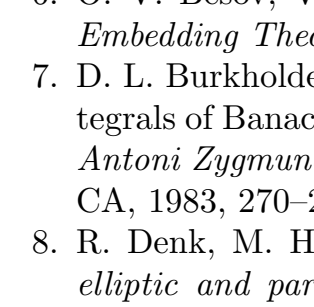

Banach J. Math. Anal. 10 (2016), no. 1, 147-168

http://dx.doi.org/10.1215/17358787-3345071

ISSN: $1735-8787$ (electronic)

http://projecteuclid.org/bjma

\title{
LINEAR AND NONLINEAR DEGENERATE ABSTRACT DIFFERENTIAL EQUATIONS WITH SMALL PARAMETER
}

\author{
VELI B. SHAKHMUROV ${ }^{1,2}$ \\ Communicated by L. P. Castro
}

\begin{abstract}
The boundary value problems for linear and nonlinear regular degenerate abstract differential equations are studied. The equations have the principal variable coefficients and a small parameter. The linear problem is considered on a parameter-dependent domain (i.e., on a moving domain). The maximal regularity properties of linear problems and the optimal regularity of the nonlinear problem are obtained. In application, the well-posedness of the Cauchy problem for degenerate parabolic equations and boundary value problems for degenerate anisotropic differential equations are established.
\end{abstract}

\section{IntroduCtion, NOTATION, AND BACKGROUND}

Boundary value problems (BVPs) for abstract differential equations (ADEs) have been studied extensively by many researchers (see [1], [3], [4], [8]-[21], and [24] and the references therein). A comprehensive introduction to ADEs and historical references may be found in [13] and [24]. The maximal regularity properties of ADEs have been studied in [1], [3]-[5], [8], [9], [16]-[20], and [23], for example. The main objective of the present article is to discuss the BVP for degenerate linear ADEs with variable coefficients

Copyright 2016 by the Tusi Mathematical Research Group.

Received Feb. 2, 2015; Accepted May 6, 2015.

2010 Mathematics Subject Classification. Primary 35J25; Secondary 35B65, 47N20.

Keywords. differential equations, semigroups of operators, Banach-valued function spaces, separable differential operators, operator-valued Fourier multipliers. 


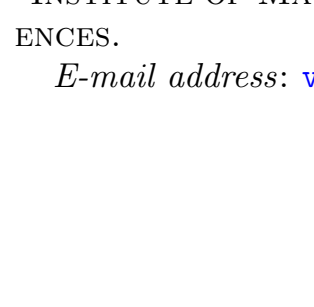

Banach J. Math. Anal. 10 (2016), no. 1, 147-168

http://dx.doi.org/10.1215/17358787-3345071

ISSN: $1735-8787$ (electronic)

http://projecteuclid.org/bjma

\title{
LINEAR AND NONLINEAR DEGENERATE ABSTRACT DIFFERENTIAL EQUATIONS WITH SMALL PARAMETER
}

\author{
VELI B. SHAKHMUROV ${ }^{1,2}$ \\ Communicated by L. P. Castro
}

\begin{abstract}
The boundary value problems for linear and nonlinear regular degenerate abstract differential equations are studied. The equations have the principal variable coefficients and a small parameter. The linear problem is considered on a parameter-dependent domain (i.e., on a moving domain). The maximal regularity properties of linear problems and the optimal regularity of the nonlinear problem are obtained. In application, the well-posedness of the Cauchy problem for degenerate parabolic equations and boundary value problems for degenerate anisotropic differential equations are established.
\end{abstract}

\section{IntroduCtion, NOTATION, AND BACKGROUND}

Boundary value problems (BVPs) for abstract differential equations (ADEs) have been studied extensively by many researchers (see [1], [3], [4], [8]-[21], and [24] and the references therein). A comprehensive introduction to ADEs and historical references may be found in [13] and [24]. The maximal regularity properties of ADEs have been studied in [1], [3]-[5], [8], [9], [16]-[20], and [23], for example. The main objective of the present article is to discuss the BVP for degenerate linear ADEs with variable coefficients

Copyright 2016 by the Tusi Mathematical Research Group.

Received Feb. 2, 2015; Accepted May 6, 2015.

2010 Mathematics Subject Classification. Primary 35J25; Secondary 35B65, 47N20.

Keywords. differential equations, semigroups of operators, Banach-valued function spaces, separable differential operators, operator-valued Fourier multipliers. 